\title{
The Role of the Pediatric Nurse Practitioner in the Comprehensive Management of Pediatric Oncology Patients in the Inpatient Setting
}

\author{
Joy Christensen, MS, RN, CPNP, and Nur Akcasu, MS, RN, CPNP
}

\begin{abstract}
The role of the pediatric nurse practitioner (PNP) in the comprehensive management of pediatric oncology patients in the inpatient setting was examined at a large tertiary teaching hospital. This article shows role responsibilities including new diagnosis teaching, procedures, routine chemotherapy, patients' comprehensive medical management, coordination of nursing care across settings, phone triage, and professional development. A PNP's typical day is highlighted to illustrate the innovative merging of traditional ambulatory care roles with that of the PNP as a comprehensive manager of pediatric oncology patients in the inpatient setting. This role provides a more seamless care experience and provides critical links in the delivery of health care to pediatric oncology patients. (1) 1999 by Association of Pediatric Oncology Nurses
\end{abstract}

$T^{1}$ HE ROLE of the primary care pediatric nurse practitioner (PNP) was created in 1965 in response to a need for increased access to ambulatory health care for children in rural settings. PNPs responded to the need for additional medical labor by performing medical skills in the primary care setting (Hravnak, Rosenzweig, \& Baldisseri, 1996). PNPs have since practiced in traditional ambulatory care settings and a variety of other settings, including tertiary care facilities. PNPs are readily available and prepared to participate in the diagnosis and management of health care problems in collaborative professional relationships and to provide coordination and continuity of medical care. Although most PNPs are traditionally educated in pri-

From the University of Michigan, C.S. Mott Children's Hospital, University of Michigan Medical Center, Ann Arbor, MI.

Address reprint requests to Joy Christensen, MS, RN, CPNP, 7 Mott Unit-based Clinical Nurse Specialist Pediatric Hematology/Oncology/Bone Marrow Transplant, University of Michigan C.S. Mott Children's Hospital, 1500 E Medical Center Dr, F 7500, Ann Arbor, MI 48109-0246.

- 1999 by Association of Pediatric Oncology Nurses. 1043-4542/99/1602-0002\$3.00/0 mary care, their assessment, diagnostic, and patient management skills are also useful in the tertiary care setting in the care of chronically ill children.

As in 1965, recent changes in the health care system and in medical education have created patient care needs that can again be addressed by PNPs. Teaching hospitals have traditionally relied heavily on residents to provide patient care coverage. Increases in primary care components in residency programs have resulted in limited acute care coverage (Hravnak et al., 1996). The recent mandate that has reduced the hours a resident may work has created a void in patient care, thus allowing PNPs to assume a more comprehensive role in managing patients in the inpatient setting. Nursing is again at a crossroads in which it can provide a much needed health care service for children who require hospitalization in a tertiary teaching facility.

The University of Michigan Medical Center is a large tertiary teaching hospital that illustrates universal changes occurring in the health care system. Pediatric education of residents has changed dramatically, result- 
ing in less resident coverage in the inpatient setting. As the University of Michigan strives to provide high-quality and more costeffective care amid changes in medical staffing, oncology PNPs have found a unique opportunity to care for patients. Since October 1992, PNPs at the University of Michigan have provided the comprehensive management of oncology patients that has been historically followed by pediatric house staff. In addition to advanced physical assessment skills, a specialized knowledge of chemotherapy and graduate education in research utilization have made PNPs ideal providers of expert inpatient care for pediatric oncology patients. The purpose of this article is to describe an advanced practice nursing role that encompasses the comprehensive management of pediatric oncology patients in the inpatient setting.

\section{Literature Review}

There are many examples in the literature of expanded roles that have been created for nurse practitioners (NPs) in response to changes in health care (Busen $\&$ Engleman, 1996; Schroer, 1991; Williams \& Valdivieso, 1994). Two articles address medical management by nurse practitioners in an ambulatory care setting. Sawyers (1993) documents medical management in the adult cancer ambulatory care setting, while Hobbie and Hollen (1993) document the outpatient medical management of childhood cancer survivors in a nurse-run late effects clinic.

Few articles discuss the role of NPs in the comprehensive management of hospitalized patients. The role of the adult NP in managing hospitalized cardiology patients has been described (Callahan, 1996). The role entailed using written standards of care similar to protocols and algorithms used in pediatric oncology. Giacalone, Mullaney, DeJoesph \& Cosma (1995) relate an example of an independent nurse-run cardiology unit. NPs admitted, cared for, and discharged adult cardiology patients on a unit run by nurses. NPs were noted to be key members of the team for the cardiac access program because of the length and level of their education, preparation in physical examination, diagnostic reasoning, and comprehensive approach to patient care management. The program was so successful that most patients were admitted within 24 hours of referral, and cardiologists requested that patients be admitted to the NP service. A pilot study conducted approximately 1 year after the inception of the program revealed that patients were satisfied with the care provided by the NPs. As primary health care providers, the advanced practitioners effectively delivered lower-cost and higher-quality care.

The role of the neonatal nurse practitioner (NNP) in the collaborative medical management of neonates in the inpatient setting is well established (Liljeblad, 1993). Liljeblad (1993) described the NNP's role in meeting the needs of the chronically ill infant by providing consistent, quality medical care and assisting in the long-term management and discharge planning. The role provided a more holistic approach to care through the continual evaluation of treatment plans and involvment of the family in discharge planning for the infant. This function of the NNP is similar to the PNP role in oncology at the University of Michigan. The oncology population is a long-term, chronically ill population cared for through an organized collaborative process. The PNP role involves individualized, episodic care with continuity provided to the child and family from hospitalization to discharge to the home and follow-up.

No information was identified that addressed the comprehensive medical and nursing management of pediatric oncology patients by PNPs in either inpatient or outpatient settings. The role most similar to that developed at the University of Michigan is the role of the PNP at Children's Medical Center of Dallas (Dale, 1991). PNPs who were once managed by resident physicians provide the medical care for patients on a pediatric surgical unit. Similar responsibilities include writing admission orders based on protocols, comprehensive medical care, and consultation with other services. For the PNPs at Children's Medical Center of Dallas, however, the inpatient and outpatient roles remain separate. PNPs at the University of Michigan are thus in the unique position of 
providing care across inpatient and outpatient settings.

\section{Role Responsibilities}

In 1992, PNPs at the University of Michigan expanded their role to include the comprehensive management of routine chemotherapy patients in the inpatient setting. Interns rotating monthly through the pediatric oncology service could not attain the level of knowledge and skills needed to effectively manage chemotherapy patients. Oncology PNPs were thus in an ideal position to provide comprehensive management in the inpatient setting. The PNP's experience in physical assessment and diagnosis and treatment, which is enhanced by the nursing emphasis on therapeutic and educational skills, offers anticipatory guidance, medical management, and advocacy to oncology patients. The integration of these skills combined with the knowledge of the health care system makes PNPs ideal providers of optimal care for the oncology patient (Callahan, 1996).

Recognizing the role PNPs could play in providing this service in the inpatient setting for the oncology population, a role definition was developed and implemented. Initially, PNPs followed a few patients who were receiving routine chemotherapy with close supervision by the attending physician. Physicians were concerned about whether PNPs had the knowledge base required to comprehensively manage routine chemotherapy patients in the inpatient setting. Therefore, PNPs had to establish credibility with physicians regarding clinical skills, critical decision making, and judgment. High visibility, meticulous attention to all aspects of medical and nursing care, and interaction with all members of the health care team helped establish the credibility of the role. As PNPs became more competent in the comprehensive management of hospitalized patients, their role became more autonomous. Today, PNPs provide comprehensive management of routine chemotherapy patients and are integral members of the oncology team in caring for the hospitalized oncology patient. Through close collaboration, physicians have seen how the role of the PNP has complemented the physi- cian role by adding a unique nursing focus to traditional medical management.

Another challenge for PNPs to overcome was the development of relationships with other specialties and caretakers. Although PNPs had carefully prepared for this new role, it was unfamiliar to staff nurses and other departments. For example, staff nurses were unaware that they could accept verbal medical orders from PNPs, and the department of pharmacy was unsure whether they could honor chemotherapy orders that were written by PNPs. A negotiated agreement had previously been made with the institution, and reviewing the components clarified the PNPs scope of practice.

The role now receives support from administration, nursing, and medical staff at the University of Michigan. PNPs play an integral role in every aspect of care across the health/ illness continuum from initial diagnosis through the course of treatment including long-term follow-up. The following describes the role of PNPs in the comprehensive management of pediatric oncology patients in the inpatient setting at the University of Michigan.

\section{New Diagnosis Teaching}

The diagnosis of cancer produces unfamiliar change for children and families. Families are plunged headlong into the bewildering world of a life-threatening illness and often aggressive treatment. PNPs begin a relationship with the family at the initial diagnosis of the illness. The initial meeting with the parents includes the oncology fellow, the PNP, the primary nurse, and the social worker. At this time, the provisional diagnosis, intended treatment, and prognosis are discussed. The PNP plays an invaluable role at this point in these family's lives. PNPs are often the primary source of information and assurances to the family that their child is receiving quality care. The rapport established during the initial weeks of treatment set the tone for a potentially long, positive relationship with the child and family members (Brown, 1989; Thoma, Hockenberry-Eaton, \& Kemp, 1993).

One of the major components of the role of the oncology PNP is new diagnosis teaching. 
New diagnosis teaching consists of measures to assist and guide the child with cancer and the family in the coping and adaptation process. New diagnosis teaching involves providing information regarding the pathophysiology of the disease, "survival skills" (e.g., when they should come to clinic, a calendar of the treatment plan, a review of blood counts, when to call, pertinent phone numbers), and side effects of cancer treatment. This initial teaching typically occurs over several sessions and may include a visit or tour of the outpatient clinic.

\section{Procedures}

Children receiving chemotherapy are faced with numerous and often painful procedures during their treatment. Since 1976, PNPs at the University of Michigan have been performing diagnostic and therapeutic procedures including bone marrow aspirations, biopsies, and lumbar punctures. Historically, PNPs performed procedures only on patients in the ambulatory care clinic. Over the years, however, PNPs have performed procedures for oncology patients in a variety of settings including inpatient units, operating rooms, and radiology and outpatient clinics. PNPs perform these procedures on a regular basis, so they are performed skillfully and expeditiously.

As autonomy and hospital-wide acceptance of the role of the PNP in the comprehensive management of routine chemotherapy patients in the inpatient setting has grown, so has the scope of the PNPs practice in performing procedures. Traditionally, procedures had been performed on conscious patients by using only local sedation without complete pain control. PNPs and anesthesiologists in a team effort have been able to provide complete pain control by using general anesthesia for many procedures. An area in the recovery room is now reserved one afternoon per week for procedures requiring general anesthesia. PNPs schedule patients in conjunction with the anesthesia service for diagnostic and therapeutic procedures such as bone marrow biopsies and lumbar punctures with intrathecal chemotherapy administration. This has provided a valuable service to children who require numerous painful procedures by minimizing the associated pain and stress.

\section{Comprehensive Management of Routine Chemotherapy Patients in the Inpatient Setting}

For PNPs to function as comprehensive managers of chemotherapy patients, it was necessary to develop a negotiated agreement between PNPs, physicians, and the nursing director. The purpose of the agreement was to establish protocols that allow PNPs to write orders in the following classifications: chemotherapeutic medications, antiemetic medications, intravenous fluids, administration of blood products, antibiotics, electrolyte replacement, antifungal agents, and parenteral nutrition. Although PNPs currently do not have prescriptive authority in the state of Michigan, the negotiated agreement allows PNPs to practice according to the established protocols.

As part of the negotiated agreement, protocols for standards of care had to be developed. In many ways, pediatric oncology is the ideal specialty to use advanced practice nursing roles because of the protocol-driven nature of treatment. The University of Michigan is a member of the Children's Cancer Group (CCG), which provides chemotherapy protocols for pediatric oncology patients. Many patients are randomly assigned to a specific protocol treatment developed by CCG. These protocols specify chemotherapy doses, intravenous fluids, supportive care, and ongoing diagnostic studies. PNPs at the University of Michigan have also been involved in writing preprinted orders for use in the inpatient setting. Preprinted orders derived from the protocols provide a template for the medical and nursing staff to follow, further ensuring compliance with CCG regulations and consistency of care for oncology patients.

CCG protocols also address treatment plans for those children who develop complications from chemotherapy. These complications include nausea and vomiting, electrolyte disturbances, fluid intolerance, nutritional complications, and hematological changes. 
PNPs collaborate with the attending physician and make appropriate nutrition, physical therapy, and social work referrals as needed to provide total care to the patient. If the medical management needs of the patient require acute care that may be beyond the PNPs scope of practice, the attending physician and PNP mutually decide to transfer care to physician coverage.

Increased medical and nursing management responsibilities have actually enhanced the advanced practice role. Within the last 2 years, PNPs have initiated the concept of primary nursing at the nurse practitioner level. For consistency, each patient is assigned a primary PNP at the time of initial diagnosis. PNPs provide the initial teaching and coordinate care across the inpatient and outpatient setting. With their extensive knowledge of this chronically ill population and the associated frequent hospitalizations, PNPs are consistently available to provide comprehensive management during transitional periods such as the monthly change of attending physician and resident physician coverage. The consistency of care provided has been very well received by patients and families. The ability of the primary PNP to follow oncology patients across the inpatient and outpatient units lends a more holistic approach to care and allows PNPs to better advocate for patients. This expanded role enhances communication with the health care team across settings and is a step further along the road to seamless health care.

\section{Outpatient Management: \\ The Traditional Role}

PNPs have traditionally functioned in the ambulatory care setting (Dale, 1991). Each PNP works 1 day per week in the ambulatory care clinic. The ambulatory care role includes following an individualized primary caseload, completing history and physical examinations, evaluating potential treatment toxicities, performing invasive procedures, writing admission and chemotherapy orders, and providing routine follow-up for patients. PNPs assure continuity of care across the health/illness continuum, including a broad spectrum of services from prevention to tertiary care.

Another facet of outpatient management is telephone triage and follow-up. PNPs return phone calls from families regarding their child's care and review all blood counts that are faxed from area labs for possible intervention. PNPs monitor patient blood counts and make medication (e.g., granulocyte stimulating factor) adjustments based on those counts. Occasionally, PNPs determine that a child needs further medical evaluation and may independently make the decision to admit the child to the hospital as part of the negotiated agreement. Because the patient's primary PNP handles all pertinent phone calls, continuity of patient care is assured.

Outpatient management also includes corresponding with local physicians. PNPs dictate and provide the pediatrician with a copy of the hospital discharge summary. Through telephone conversations, PNPs assist in the transition of the patient and family's return to their local community by keeping the local pediatrician updated regarding ongoing discharge problems and necessary follow-up. PNPs provide education to the local pediatrician regarding any required outpatient chemotherapy that will be administered in the local pediatrician's office. This function includes educating the nursing staff on administration and safety issues. PNPs also provide support and information on primary care issues such as immunization guidelines and sports restrictions. All of these measures insure continuity of care and consistency of information given to families.

\section{Education}

The educational component of the PNP role involves both formal and informal in: struction. Formally, PNPs are content experts and provide classes to nurses in chemotherapy, basic oncology and advanced oncology. PNPs also facilitate the educational process of graduate nursing students who wish to become advanced practice nurses. For physicians, PNPs have authored chapters in the fellow orientation handbook on a variety of topics such as central line 
catheters and the management of patients with fever and neutropenia.

Informally, PNPs are nurse and physician resources. For nurses, PNPs assist in trouble shooting, problem solving, and participation in the collaborative management of chemotherapy issues. PNPs act as role models for oncology nurses by being strong advocates for patients and families. For physicians, PNPs provide ongoing teaching and consultation by being available resources in the dayto-day care of pediatric oncology patients.

\section{Professional Development}

Professional development is an integral part of the role of the PNP in pediatric oncology. The ever-changing health care environment and advances in the profession require PNPs to remain current in their specialty. Integration of theory, research, and practice serves to advance the PNP knowledge base and fosters a current clinical base of practice. PNP involvement and professional development is noted institutionally, locally, and nationally.

Institutionally, PNPs participate in a multidisciplinary monthly journal club that regularly reviews research pertaining to clinical oncology. In addition, they review and present nursing research in oncology to their physician colleagues each quarter. PNPs participate in the local chapter of the Association of Pediatric Oncology Nurses. For many years, they have served as officers or guest lecturers for educational meetings. This affiliation with professional organizations affords the opportunity to maintain bonds and share ideas with nursing peers, which contributes to a more collegial atmosphere. PNPs are also members of national PNP organizations and oncology nursing organizations. They strive for institutional representation at all relevant national conferences. Furthermore, PNPs are involved in CCG committees to participate in the collection of data to assist in the development of better cancer treatments for children. Participation in these organizations serves to advance nursing knowledge in oncology and to maintain a contemporary clinically based practice.

\section{Rewards of the Expanded Role}

PNPs at the University of Michigan are responsible for the overall health care management of oncology patients from admission to discharge. Functioning in such an autonomous role with the ultimate responsibility for clinical effectiveness can be both challenging and rewarding. The most rewarding component of the expanded role is the nursing qualities of the role (Callahan, 1996). This role allows PNPs to enhance their clinical skills and still maintain a high degree of patient contact. The primary nurse practitioner role allows the PNP to be a resource and problem solver and allows for collaborative management as the patient and family move through the health care experience. The strong bond that develops among the patient, the family, and the PNP is one of the most rewarding aspects of the role.

\section{A Typical Day}

A typical day for the oncology PNP begins at 8:00 a.m. The PNP discusses any patient issues or problems that have occurred overnight with the on-call physician. This step is followed by a discussion regarding the patients on the unit with the other nurse practitioners. The workload is already determined by the primary inpatient caseload of each nurse practitioner.

Typically, there are up to 10 inpatients receiving routine chemotherapy. The PNP reviews bedside documentation and the events of the past 12 hours including discussion and input from the staff nurses. This review includes the patients' vital signs, chemotherapy drugs, administration times, intake and output, and antiemetic usage. The review of documentation includes a discussion with the nurse who is caring for the patient to address any additional issues. Typical issues discussed include the patient's response to chemotherapy, supportive care measures such as pain control, effectiveness of antiemetics, financial concerns, cultural concerns, and sibling and family coping issues.

Before the daily routine physical examination and assessment, the PNP reviews perti- 
nent laboratory data and diagnostic test results. The physical assessment includes an examination focusing on the systems most acutely affected by chemotherapy, because a thorough physical and history were obtained upon admission. At the time of examination, the PNP listens to the patient and family, addresses any questions or concerns, and provides patient and family teaching.

One PNP attends daily multidisciplinary rounds, which includes the attending physician, an oncology fellow, one resident, two interns, a PNP, a social worker, the unitbased clinical nurse specialist (CNS), and a home care representative. The PNP reports on all inpatient chemotherapy patients and receives input on the plan of care from the other disciplines. The PNP informs the team of the patients' progress including any necessary referrals. The role of the PNP in multidisciplinary rounds is not only that of inpatient medical manager of routine chemotherapy patients but also that of an expert resource to all patients on the oncology service.

Throughout the day, the PNP is responsible for performing diagnostic or routine bone marrow aspirations, biopsies, and lumbar punctures including consultations from other disciplines. After rounds, a review of the chart is completed, progress notes are written, necessary diagnostic tests are ordered, orders are updated, referrals are made, and required transfusions are ordered.

Patients receiving chemotherapy are admitted from the oncology clinic after outpatient evaluation. The outpatient PNP completes the assessment and chemotherapy orders. The inpatient PNP co-signs the chemotherapy orders after recalculating dosages. Only PNPs, oncology fellows, and attending physicians may co-sign chemotherapy orders. PNPs are familiar with the protocols and complete the admission paperwork quickly. This system decreases the time families have to wait for chemotherapy and provides a more seamless admission experience.

After co-signing the orders, the PNP ensures that all required diagnostic tests are completed at the appropriate intervals according to the protocol. The chart is re- viewed, with the PNP incorporating the patient's past history and response to chemotherapy. An admission progress note is written and discharge planning is initiated, because many patients only require an overnight admission.

The afternoon is dedicated to a variety of functions, including new diagnosis teaching, returning outside phone calls, writing discharge summaries, completing discharge paperwork, and dictation. The PNPs arrange appropriate follow-up care including diagnostic tests, clinic appointments, and preparation for the next admission for all hematology/ oncology patients. Often, this work involves coordination with outside community services such as pharmacies, other hospitals, and physician's offices.

At the end of the day, an updated summary on each patient is given to the on-call resident of the pediatric oncology service. Specific patient care issues are identified and interventions are clearly outlined to assure continuity of the established plan of care.

\section{Future Directions}

In the future, PNPs at the University of Michigan Oncology Department will have additional opportunities to expand their role. With the opening of a new cancer center, the pediatric oncology clinic will include expanded evening hours. This practice will provide an opportunity for PNPs to clinically triage oncology patients in the clinic setting, thereby decreasing the need for evaluation in the emergency room.

With even less resident coverage being proposed for the upcoming year, PNPs will have an opportunity to comprehensively manage a broader patient population such as hospitalized patients with fever and neutropenia and patients requiring blood product transfusions. As the PNPs develop a larger knowledge base and expanded practice skills, they can undertake the care of patients requiring more complex decision making.

The identification and testing of specific outcome criteria is critical to evaluate the role of the PNP. These should reflect both the tangible and intangible aspects of the role. 
There are several possible outcome measures that are well documented in the literature: average length of stay, revenue generated, documentation of care delivery, clinic waiting time, patient satisfaction with care, reduction of time spent by physicians on procedures, discharge summaries, and outpatient clinic visits (Ingersoll, 1995). There is currently no formal evaluation process of the impact of the role of the PNP as comprehensive health care manager. This lack of an evaluation process will be the subject for a research project for the PNPs in the future.

The enhanced role of PNPs as direct providers and prescribers of care to the individual patient and family provides a comprehensive approach to patient-focused care. Regardless of the setting, PNPs advanced practice roles are characterized by the predominance of self-initiated treatment protocols rather than dependent functions. The ability of PNPs to practice across settings has lent a more seamless care experience and has provided a critical link in the delivery of health care to oncology patients.

\section{References}

Busen, N.H., E Engleman, S.G. (1996). The CNS with practitioner preparation: an emerging role in advanced practice nursing. Clinical Nurse Specialist, 10(3), 145 150.

Brown, P.G. (1989). Families who have a child diagnosed with cancer: what the medical caregiver can do to help them and themselves. Issues in Comprehensive Pediatric Nursing, 12, 247-260.

Callahan, M. (1996). The advanced practice nurse in an acute care setting: the nurse practitioner in adult cardiac surgery care. Nursing Clinics of North America, 31(3), 487-493

Dale, J. (1991). New role for PNPs in an inpatient setting. Journal of Pediatric Health Care, 5(6), 336-337.

Giacalone, M.B., Mullaney, D., DeJoesph, D.A., $\mathcal{E}$ Cosma, M. (1995). Practice model: development of a nurse managed unit and the advanced practitioner role. Critical Care Nursing Clinic of North America, 7(1), 35.41

Hobbie, W.L., \& Hollen, P.J. (1993). Pediatric nurse practitioners specializing with survivors of childhood cancer. Journal of Pediatric Health Care, 7(1), 24-30.

Hravnak, M., Rosenzweig, M.Q., $\&$ Baldisseri, M. (1996). Current questions with regard to acute care
In preparation for the future, we must examine the past. As we once again reach a crossroads in the ever changing health care system, pediatric nurse practitioners are seizing advanced practice opportunities and forging new roles for the profession. The innovative merging of the traditional ambulatory care role with that of PNPs as comprehensive health care managers provides increased professional leadership and autonomy. This advanced practice role is one pediatric oncology department's solution to the current health care environment.

\section{Acknowledgments}

A special thanks to Judy Cameron PhD, RN, CPNP and Marilyn Svejda PhD, RN from the University of Michigan School of Nursing for their support, guidance, and high standards. In addition, I would like to thank Marcia Leonard, CPNP and Judy Moyer MS, CPNP for their memory for detail and their commitment and dedication to all the pediatric oncology patients cared for at the University of Michigan Mott Children's Hospital.

nurse practitioner preparation and role implementation. AACN Clinical Issues, 7(2), 289-299.

Ingersoll, G. (1995). Evaluation of the advance practice nurse role in acute and specialty care. Critical Care Nursing Clinics of North America, 7(1), 25-33.

Liljeblad, C.Y. (1993). Neonatal nurse practitioners: paving the way for case management of chronically ill infants and their families. Journal of Perinatal Neonatal Nursing, 7(3), 49-58.

Sawyers, J.E. (1993). Defining your role in ambulatory care: clinical nurse specialist or nurse practitioner? Clinical Nurse Specialist, 7(1), 4-7.

Schroer, K. (1991). Case management: clinical nurse specialist and nurse practitioner, converging roles. Clinical Nurse Specialist, 5(4), 189-194.

Thoma, M.E., Hockenberry-Eaton, M., $\varepsilon$ Kemp, V. (1993). Life change events and coping behaviors in families of children with cancer. Journal of Pediatric Oncology Nursing, 10, 105-111.

Williams, C.A., \& Valdivieso, G.C. (1994). Advanced practice models: a comparison of clinical nurse specialist and nurse practitioner activities. Clinical Nurse Specialist, $8(6), 311-318$ 